\title{
Unilateral submandibular gland aplasia with ipsilateral sublingual gland hypertrophy presenting as a neck mass
}

\author{
Gonzalo Herrera-Calvo ${ }^{1}$, Belén García-Montesinos-Perea ${ }^{2}$, Ramón Saiz-Bustillo ${ }^{3}$, Jaime Gallo-Terán ${ }^{4}$, Pe- \\ dro Lastra-García-Barón ${ }^{5}$
}

\author{
${ }^{1}$ Oral and Maxilofacial Surgery Department. Hospital Universitario Marqués de Valdecilla, Santander (Cantabria, Spain). Resi- \\ dent \\ ${ }^{2}$ Oral and Maxilofacial Surgery Department. Hospital Universitario Marqués de Valdecilla, Santander (Cantabria, Spain). At- \\ tending Surgeon \\ ${ }^{3}$ Oral and Maxilofacial Surgery Department. Hospital Universitario Marqués de Valdecilla, Santander (Cantabria, Spain). Head \\ of Department \\ ${ }^{4}$ Deparment of Radiodiology. Hospital Universitario Marqués de Valdecilla, Santander (Cantabria, Spain). Resident \\ ${ }^{5}$ Deparment of Radiodiology. Hospital Universitario Marqués de Valdecilla, Santander (Cantabria, Spain). Staff radiologist
}

Correspondence:

Hospital Universitario Marqués de Valdecilla

Servicio de Cirugía Oral y Maxilofacial

Avenida de Valdecilla 25

39008 Santander (Cantabria) Spain

gherreracalvo@gmail.com

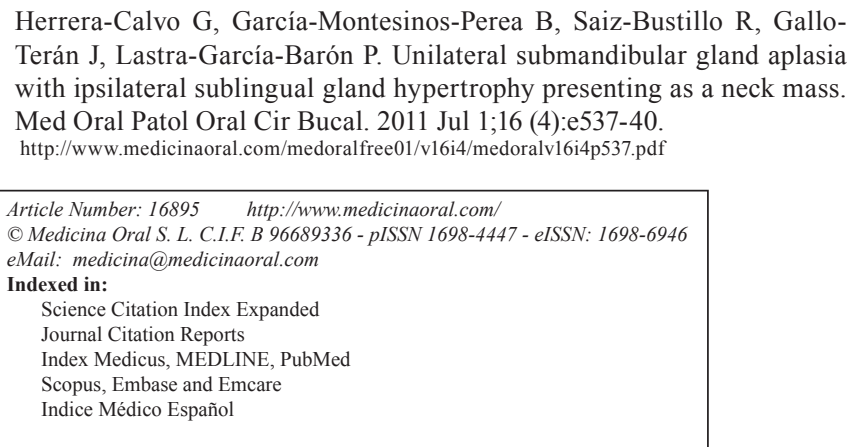

\begin{abstract}
The congenital absence of the major salivary glands is a very infrequent disorder, in which several glands are usually involved at the same time. Sometimes this disorder can be associated with other developmental anomalies. The unilateral aplasia of the submandibular gland is an extremely rare finding with only 14 cases reported in the literature. Clinically, this kind of patients may complain of dryness of the mouth, difficulties in chewing and swallowing, severe periodontal disease or multiple caries, but usually they follow an asymptomatic course. Salivary gland aplasia can be diagnosed with a large variety of imaging techniques, which include computer tomography (CT), magnetic resonance imaging (MR), ultrasonography (US), sialography, or scintigraphy. In this paper we report a case of a patient referred to our department with a long term and progressive growing neck mass, who has an unilateral submandibular gland aplasia associated to an ipsilateral hypertrophy of the sublingual gland.
\end{abstract}

Key words: Submandibular gland aplasia, sublingual gland hypertrophy, neck mass. 


\section{Introduction}

Major salivary gland agenesis is a very uncommon disorder. Since the first case described in 1885, about 40 cases have been reported in the literature. Most of them were a congenital absence of all major salivary glands. On the other hand, unilateral submandibular gland aplasia is an extremely rare disorder. According to our review, only 14 cases have been described. Most of them were incidental findings because of the lack of symptoms. In this study we report a case of an unilateral submandibular gland aplasia with ipsilateral hypertrophy of the sublingual gland in a patient complaining for a neck mass.

\section{Case Report}

A 26-year-old woman without any relevant past medical or surgical history was referred to our department because of a long term submandibular swelling with progressive growth. The patient didn't complain about

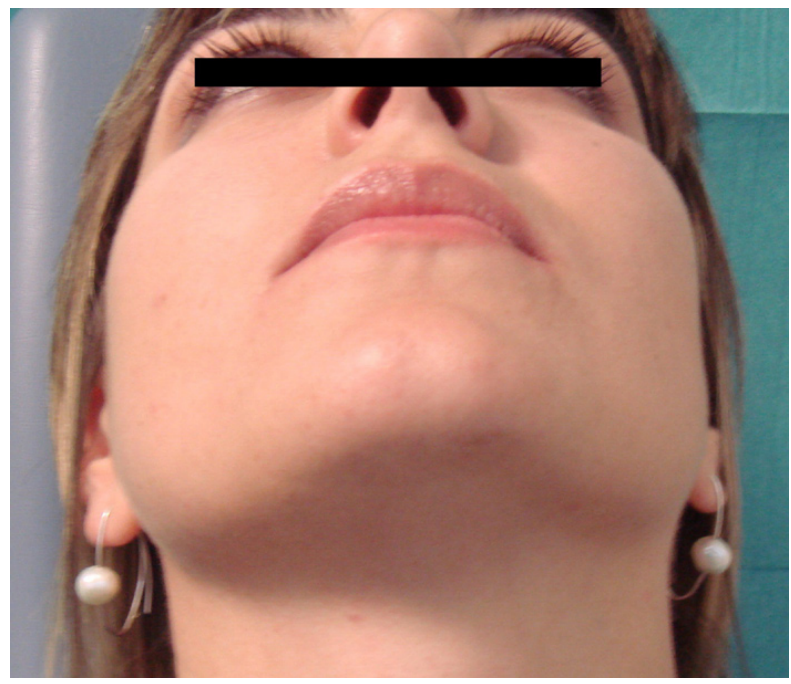

Fig. 1. Soft tissue enlargement in the right submandibular area.

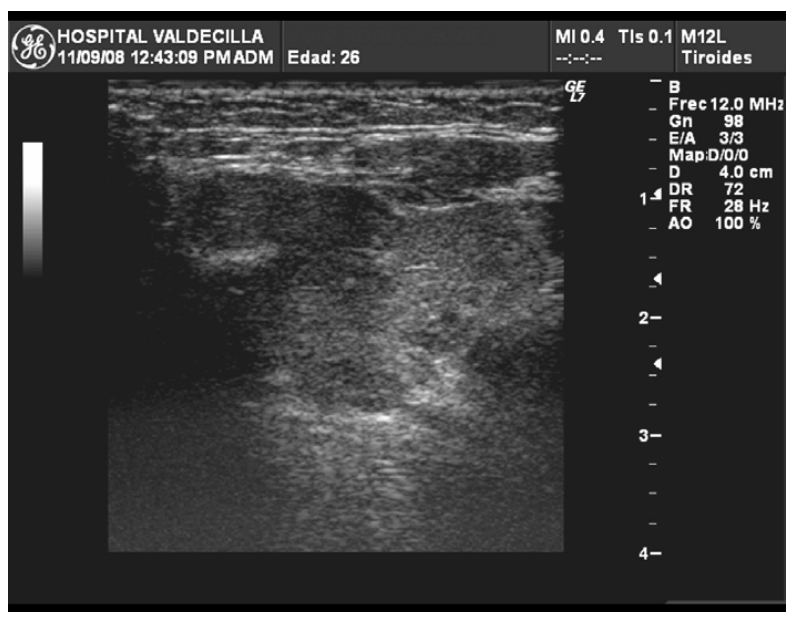

Fig. 2. Ultrasonography of the right sublingual space (coronal cut) Sublingual gland hypertrophy. any symptomatology, apart from a significant anxiety produced by the increasing size of the mass and the ignorance of her pathology.

Inspection showed an asymmetry in the submandibular region, due to a soft tissue enlargement at the right side (Fig. 1), as well as some ipsilateral floor of the mouth elevation. Extraoral palpation was unremarkable, but intraorally there was a lobulated painless and mobile soft mass of about 2 centimetres in the right floor of the mouth. Oral mucosa had no alterations. The rest of oropharyngeal and cervical examination was normal.

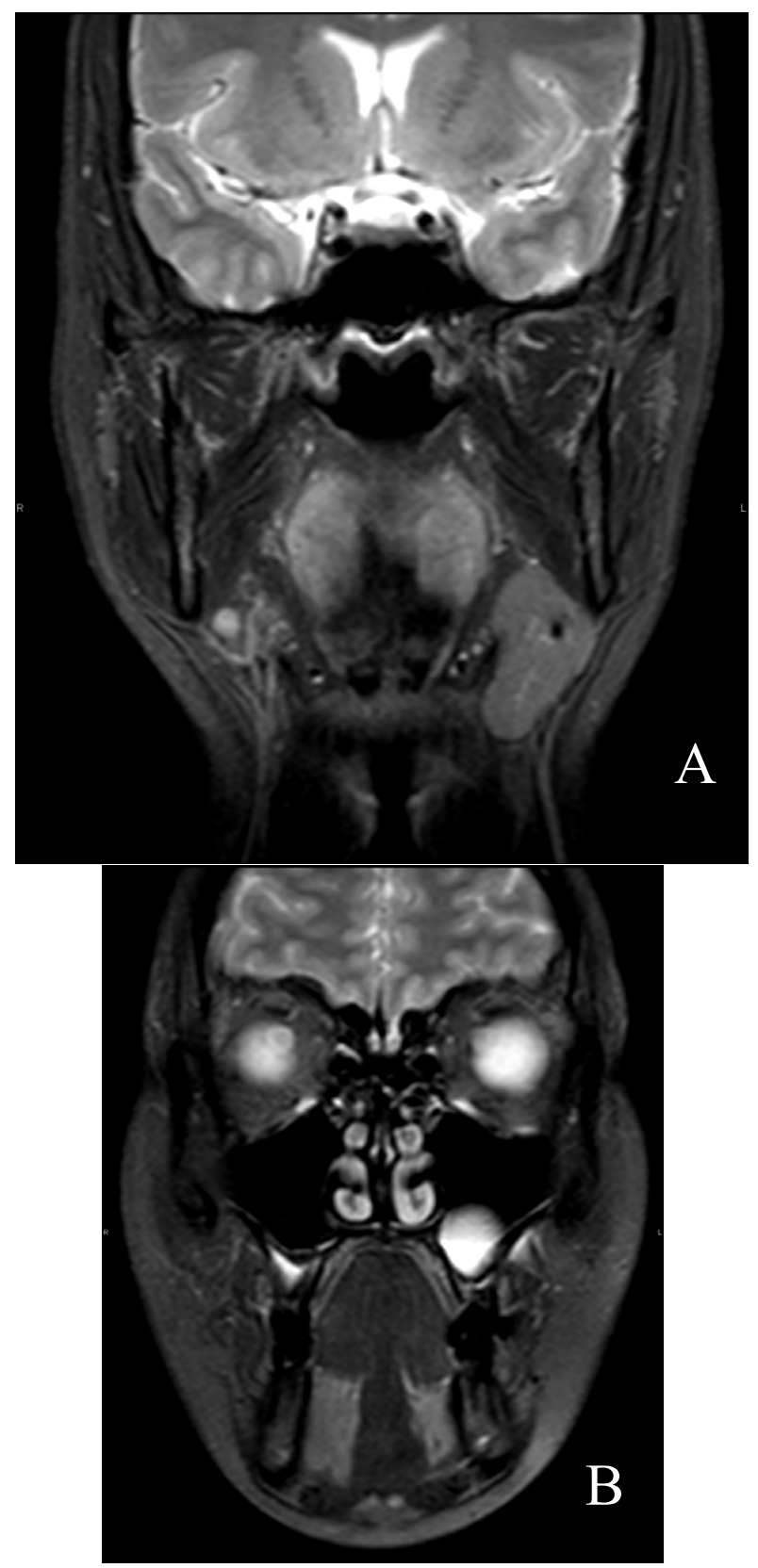

Fig. 3. T2-weightened images of magnetic resonance (coronal cuts) A, Right submandibular gland absence. B, Right sublingual gland hypertrophy. 
Neck US showed a slightly hyperechogenic homogeneous solid mass with smooth margins, located in the right sublingual space (Fig. 2). No calcifications or areas of necrosis were noted. The right submandibular gland could not be identified. No anomalies were observed in the remaining major salivary glands, and cervical adenopathies were absent. These findings were compatible with a hypertrophied right sublingual gland in association with the absence of the ipsilateral submandibular gland.

Our study was completed with MR. Axial and coronal $\mathrm{T} 1$ and T2-weighted images showed absence of the right submandibular gland and an enlarged right sublingual gland, with the same signal characteristics as observed in the contralateral sublingual gland. Right sublingual gland measured 22'38 x 12'47 millimetres, while the left one measured 16'39 x 7'27 millimetres. Both parotid glands and the left submandibular gland were normal, as well as the rest of the study (Fig. 3).

\section{Discussion}

Salivary glands arise sequentially between the fourth and the eighth week of fetal development as solid epithelial buds from primary oral cavity, which grow and extend into the underlying mesenchymal tissue as a solid core of cells, which then undergoes extensive branching and eventually develops lumina. Parotid glands appear during de fourth week of gestation, submandibular glands during the sixth, and sublingual glands during the ninth (1).

The etiology of major salivary glands aplasia is still unknown. This entity can accompany other developmental anomalies, such as Treacher-Collins syndrome, hemifacial microsomia, cleft palate or absence of the lacrimal puncta $(2,3)$. These abnormalities suggest that this entity may be associated with ectodermal defects of the first and second branchial arches and may be the result of some disturbing influence in early fetal development (4). However congenital unilateral absence of the submandibular gland is not associated with other developing anomalies, as well as our patient, which suggests that this is an isolated phenomenon derived from other unique defect of fetal development (5).

Clinical manifestations of the patients with salivary gland aplasia can vary depending on the number of glands affected, their contribution in the salivary flow production, and the presence of compensatory hypertrophy of the other glands. Some of them may be asymptomatic, while others may present dryness of the mouth, chewing and swallowing difficulties or an increased incidence of dental caries. This fact is thought to be due to reduction of the protective effect of saliva within the oral cavity (2).

Most of the patients with unilateral submandibular gland aplasia reported in the literature had no symp- toms related to the salivary gland absence, as well as our case. Only two cases had dry lips and mouth, difficulties with chewing solid foods and changes in taste $(6,7)$. A neck mass appearance as first manifestation has been only described three times, in two of them because of a compensatory gland hypertrophy (8), and in the other case because of a pleomorphic adenoma of the contralateral submandibular gland (9). Unilateral submandibular masses can be attributed to a high number of pathologies with several origins. In the case described by Shipchandler y Lorenz (8) the patient had a history of squamous cell carcinoma of the tongue ipsilateral to the mass, so the differential diagnosis was first established with a metastatic lymphadenopathy. Our patient had no history of oral cavity carcinoma. Other diagnostic possibilities considered were chronic sialadenitis, inflammatory lymphadenopathy, salivary gland tumor and lipoma.

Salivary gland aplasia can be diagnosed with a variety of imaging techniques, which include CT, MR imaging, US, sialography or scintigraphy $(2,3,10)$. US is an ideal tool for initial assessment of lesions in the superficial parotid and submandibular gland. However, lesions in the sublingual gland, located deeper and with a higher percentage of malignant tumors, should be evaluated by MR imaging (11). CT cannot successfully differentiate the sublingual gland from the neighbouring muscles. However, the ability of MR imaging to differentiate the sublingual gland from the surrounding tissues facilitates the differential diagnosis of the lesions in this space. The sublingual gland has intermediate T1weighted signal intensity, lower than the fat and higher than the muscle. On T2-weighted images the sublingual gland remains hyperintense to muscle. Scintigraphy has been described in some cases in the literature $(2,3,12)$, but although this is a good method for determining the functional activity of the salivary glands, it doesn't define their anatomy (9). This technique may be useful for discarding malignant origin in patients with personal history of malignant tumors, as well as the fine needle aspiration biopsy (FNA) (8). Sialography by cannulating Wharton's duct has also been used for submandibular gland agenesis confirmation (5). In our patient, US was used as first imaging technique showing the right submandibular gland aplasia; this ecographyc findings were confirmed by MR imaging, which demonstrated the ipsilateral sublingual gland hypertrophy.

The assessment of the patient and his family is essential, and follow up seems to be worthwhile. Some authors think that transoral sublingual gland excision should be considered in cases where the swelling were aesthetically bothersome. In our case, the diagnosis and the exclusion of other pathologies was enough for the patient, who refused any therapy.

In conclusion, unilateral submandibular gland aplasia 
is an extremely rare disorder, and its real incidence is probably unknown. Careful evaluation of the patients who complain of xerostomy, chewing and swallowing difficulties, severe periodontal disease and a neck mass, may lead to the detection of new cases of this entity. US and MR imaging are adequate techniques for this patient's study.

\section{References}

References with links to Crossref - DOI

1. Johns ME. The salivary glands: anatomy and embryology. Otolaryngol Clin North Am. 1977;10:261-71.

2. McDonald FG, Mantas J, McEwen CG, Ferguson MM. Salivary gland aplasia: an ectodermal disorder? J Oral Pathol. 1986;15:115-7.

3. Higashino H, Horii T, Ohkusa Y, Ohkuma H, Ino C, Nakazawa $\mathrm{M}$, et al. Congenital absence of lacrimal puncta and of all major salivary glands: case report and literature review. Clin Pediatr (Phila). 1987;26:366-8

4. Kubo S, Abe K, Ureshino T, Oka M. Aplasia of the submandibular gland. A case report. J Craniomaxillofac Surg. 1990;18:119-21.

5. Roh JL. Unilateral submandibular gland aplasia: an isolated phenomenon of early fetal development. Otolaryngol Head Neck Surg. 2006;135:332-4.

6. Yilmaz M, Karaman E, Isildak H, Enver O, Kilic F. Symptomatic unilateral submandibular gland aplasia associated with ipsilateral sublingual gland hypertrophy. Dysphagia. 2010;25:70-2.

7. Gallego L, Junquera L, Cuesta P, Rosado P. Symptomatic unilateral submandibular gland aplasia. Br J Oral Maxillofac Surg. 2009:47:243.

8. Shipchandler TZ, Lorenz RR. Unilateral submandibular gland aplasia masquerading as cancer nodal metastasis. Am J Otolaryngol. 2008;29:432-4.

9. García-Consuegra L, Gutiérrez LJ, Castro JM, Granado JF. Congenital unilateral absence of the submandibular gland. J Oral Maxillofac Surg. 1999;57:344-6.

10. Yoshiura K, Yamada M, Yamada N. Demonstration of congenital absence of then major salivary glands by computed tomography. Dentomaxillofac Radiol. 1990;2:77-8.

11. Lee YY, Wong KT, King AD, Ahuja AT. Imaging of salivary gland tumours. Eur J Radiol. 2008;66:419-36.

12. Sucupira MS, Weinreb JW, Camargo EE, Wagner HN Jr. Salivary gland imaging and radionuclide dacryocystography in agenesis of salivary glands. Arch Otolaryngol. 1983;109:197-8. 